\title{
Analysis of Grain Boundary Migration in Alumina
}

\author{
Shelley R. Gilliss, Nicole Munoz, Christopher R. Perrey, Jessica Riesterer, N. Ravishankar ${ }^{1}$, \\ Jeffrey K. Farrer², C. Barry Carter
}

Dept of Chemical Engineering and Materials Science, University of Minnesota, 421 Washington

Ave. S.E., Minneapolis, MN 55455

${ }^{1}$ Now at Indian Institute of Science, Bangalore, India

${ }^{2}$ TSL/EDAX, Draper, UT 84020

An understanding of grain boundary migration (GBM) is of fundamental importance in ceramic materials, as liquid phase sintering is commonly used to form monolithic forms. Past studies have lead to advances in the understanding of GBM in the presence of a glassy intergranular phase in $\mathrm{Al}_{2} \mathrm{O}_{3}$ and without a glassy phase [1-4]. However, these studies rely on information obtained from an examination of the free surface of the specimen which does not give a complete picture of the motion of the grain boundary in the bulk material.

In this study a technique has been developed to study the evolution of surface grooves from migrating grain-boundaries in high-purity polycrystalline alumina and bicrystals of controlled crystallography. Visible-light (VLM), atomic-force (AFM), scanning electron (SEM) and transmission electron microscopy (TEM) in addition to electron-backscatter diffraction (EBSD) and a focused-ion beam (FIB) tool were used to characterize boundary migration in alumina. EBSD analysis was carried out on a Philips XL30 FEG SEM equipped with a DigiView 1612 high-resolution, high-speed CCD camera. A $2.5 \mathrm{~cm}$ sample-to-camera distance was used and $\sim 70^{\circ}$ sample tilt. A Philips CM30 operated at $300 \mathrm{kV}$ was used for TEM characterization, a Digital Instruments Nanoscope III, operated in contact mode was used for AFM and an FEI DB235 was used for FIB work.

Pulsed-laser deposition (PLD) has been used to deposit thin films ( $\sim 100 \mathrm{~nm}$ thick) of glass on single-crystals of alumina. The film/substrate assembly is then fabricated into bicrystals of known boundary-plane orientation by hot pressing. For polycrystalline specimens, VLM was used to map the central regions of the polycrystalline samples. Using the VLM images as a guide to revisit the same boundaries, groove profiles were examined with (AFM) following successive heat treatments and the evolution of grain morphology was monitored. AFM measurements reveal asymmetry in most of the grooves and also that faceting at the base of the groove can result in variations in groove depth measurements of up to $50 \mathrm{~nm}$ for a particular boundary. Cross-sectioned samples for TEM analysis of grain boundaries were made using FIB perpendicular to the boundary's intersection with the free surface. Viewing the cross-sectioned samples using SEM indicates that one reason for the asymmetry is the boundary's angle of inclination with the surface. EBSD patterns were taken from regions through which grainboundaries had migrated and from surrounding grains in order to correlate the magnitude of orientation mismatch to the relative distance of boundary migration.

The extent of the sub-surface migration can be analyzed by TEM after a FIB section is made at a site-specific migrated grain boundary. Figure 1 is a SEM image of a bicrystal made from singlecrystal alumina with the (0001) plane oriented at the boundary plane and high-purity polycrystalline alumina. It has been heat treated at $1650^{\circ} \mathrm{C}$ for 10 hours. At the specified location in Figure 1, the boundary has migrated approximately $7 \square \mathrm{m}$ into the polycrystalline alumina at the noted location for the FIB membrane. The use of the FIB tool reveals that the sub-surface migration is much greater than that of the surface, as can be seen in the TEM montage image of Figure 2. The initially flat interface has migrated an additional $1.8 \square \mathrm{m}$ into the polycrystalline alumina. By using this technique, it is possible to include the effect of surface grooving at the free surface in the theories of GBM by the comparison of the behavior of these grain boundaries in the bulk material. 


\section{References}

1. $\quad$ N. Ravishankar and C.B. Carter,. Microsc. Microanal., 5 (1999) (Suppl. 2) 800.

2. M. P. Mallamaci and C.B. Carter, J. Am. Ceram. Soc., 82 (1999) 33.

3. N. Munoz et al. Mat. Res. Symp. Soc. Proc. 740 (2003), in press.

4. $\quad$ S. Ramamurthy et al., Mater. Sci. Forum, (1996) 207.

5. This work was greatly aided by Jim Bentley (Oak Ridge National Laboratory), Velemir Radmilovic and Eric Stach (National Center for Electron Microscopy). This research is supported by the U.S. Department of Energy through grant DE-FG02-01ER45883.

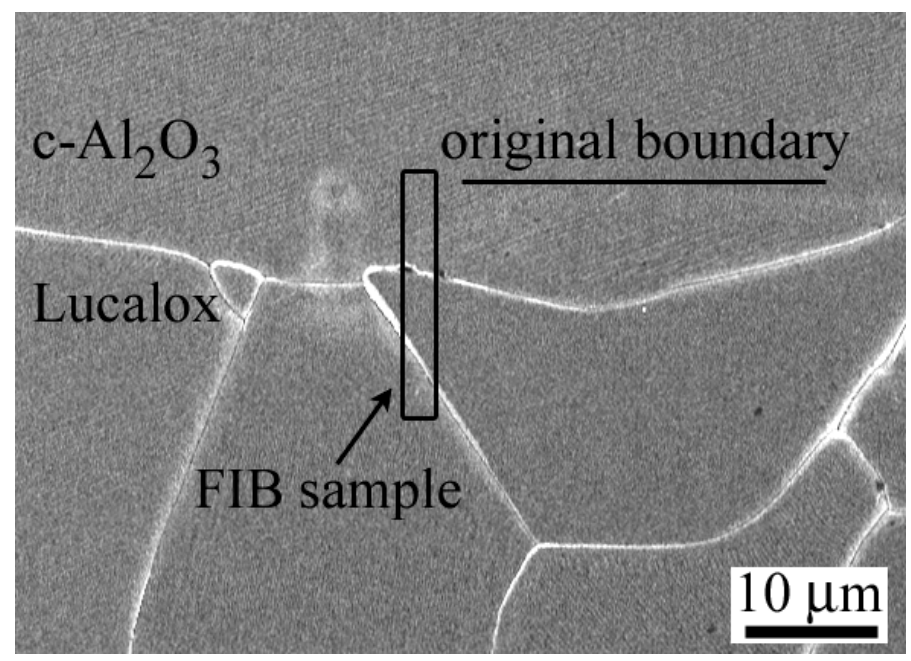

FIG. 1. SEM image of a region of a $\mathrm{Al}_{2} \mathrm{O}_{3} /$ polycrystalline alumina $\left(\right.$ Lucalox $\left.^{\mathrm{TM}}\right)$ bicrystal where the boundary has migrated $(\sim 7 \square \mathrm{m})$. The initial boundary plane on the single-crystal was the basal plane (0001). The location of the FIB-prepared TEM specimen is noted in the figure.

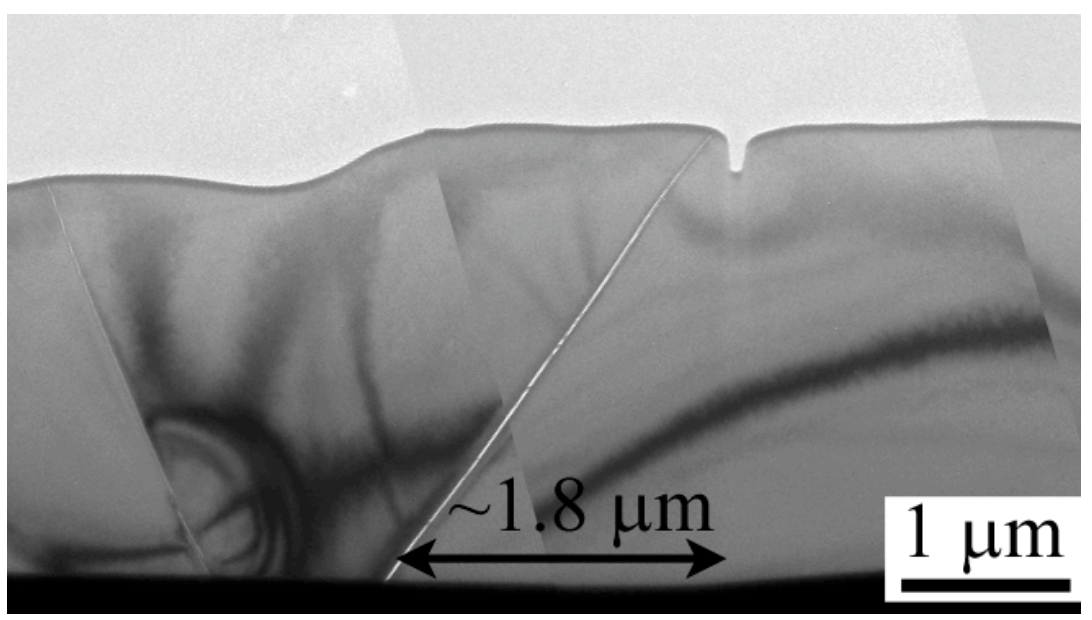

FIG. 2. TEM montage of the migrated boundary noted in Figure 1. The boundary has migrated $1.8 \square \mathrm{m}$ further into the polycrystalline region than is evident from the surface images. This additional sub-surface migration is likely due to the grooving at the surface which accommodates the lowering of the free-surface energy. 